University of Wollongong

Research Online

Faculty of Social Sciences - Papers (Archive) Faculty of Arts, Social Sciences \& Humanities

$1-1-2017$

Electroencephalogram Theta/Beta Ratio and Spectral Power Correlates of Executive Functions in Children and Adolescents With AD/HD

Dawei Zhang

University of Wollongong, dwz847@uowmail.edu.au

Hui Li

Peking University

Zhanliang Wu

Peking University

Qihua Zhao

Peking University

Yan Song

Beijing Normal University

See next page for additional authors

Follow this and additional works at: https://ro.uow.edu.au/sspapers

Part of the Education Commons, and the Social and Behavioral Sciences Commons

Research Online is the open access institutional repository for the University of Wollongong. For further information contact the UOW Library: research-pubs@uow.edu.au 


\title{
Electroencephalogram Theta/Beta Ratio and Spectral Power Correlates of Executive Functions in Children and Adolescents With AD/HD
}

\begin{abstract}
OBJECTIVE: The electroencephalogram (EEG) has been widely used in AD/HD research. The current study firstly aimed to replicate a recent trend related to EEG theta/beta ratio (TBR) in children and adolescents. Also, the study aimed to examine the value of resting EEG activity as biomarkers for executive function $(E F)$ in participants with AD/HD. METHOD: Fifty-three participants with AD/HD and 37 healthy controls were recruited. Resting EEG was recorded with eyes closed. Participants with AD/HD additionally completed EF tasks via the Cambridge Neuropsychological Test Automated Battery. RESULTS: TBR did not differ between groups; however, TBR was positively correlated with inattentive symptoms in AD/HD. Other correlations were found between EEG activity and neuropsychological functions including spatial planning and decision making in the AD/HD group. CONCLUSION: The results do not support the diagnostic value of TBR. Instead, given the heterogeneous features, the results support the prognostic value of EEG in $A D / H D$.
\end{abstract}

\section{Keywords}

electroencephalogram, theta/beta, ratio, spectral, children, power, correlates, ad/hd, adolescents, functions, executive

\section{Disciplines}

Education | Social and Behavioral Sciences

\section{Publication Details}

Zhang, D., Li, H., Wu, Z., Zhao, Q., Song, Y., Liu, L., Qian, Q., Wang, Y., Roodenrys, S., Johnstone, S. J., De Blasio, F. M. \& Sun, L. (2019). Electroencephalogram Theta/Beta Ratio and Spectral Power Correlates of Executive Functions in Children and Adolescents With AD/HD. Journal of Attention Disorders, 23 (7), 721-732.

\section{Authors}

Dawei Zhang, Hui Li, Zhanliang Wu, Qihua Zhao, Yan Song, Lu Liu, Qiujin Qian, Yufeng Wang, Steven J. Roodenrys, Stuart J. Johnstone, Frances M. De Blasio, and Li Sun 


\section{Electroencephalogram Theta/Beta Ratio and Spectral Power Correlates of Executive Functions in Children and Adolescents with AD/HD}

Da-Wei Zhang ${ }^{1}$, Hui $\mathrm{Li}^{2,3}$, Zhanliang $\mathrm{Wu}^{2,3}$, Qihua Zhao ${ }^{2,3}$, Yan Song ${ }^{4}$, Lu Liu ${ }^{2,3}$, Qiujin Qian $^{2,3}$, Yufeng Wang ${ }^{2,3}$, Steven Roodenrys ${ }^{1}$, Stuart J. Johnstone ${ }^{1}$, Frances M. De Blasio ${ }^{1}$, and Li Sun ${ }^{2,3}$

${ }^{1}$ Brain \& Behaviour Research Institute and School of Psychology, University of Wollongong, Australia ${ }^{2}$ Peking University Sixth Hospital/Institue of Mental Health, Beijing, China ${ }^{3}$ National Clinical Research Center for Mental Disorders, Beijing, China ${ }^{4}$ Beijing Normal University, Beijing, China

\section{Corresponding Author:}

Li Sun, Peking University Sixth Hospital, Beijing 100191, China.

Email: sunlioh@bjmu.edu.cn

Stuart J. Johnstone, University of Wollongong, Wollongong, New South Wales 2522, Australia

Email: sjohnsto@uow.edu.au 


\begin{abstract}
The electroencephalogram (EEG) has been widely used in AD/HD research. The current study firstly aimed to replicate a recent trend related to EEG theta/beta ratio (TBR) in children and adolescents. Also, the study aimed to examine the value of resting EEG activity as biomarkers for executive function $(\mathrm{EF})$ in participants with $\mathrm{AD} / \mathrm{HD}$. Method: Fifty-three participants with AD/HD and 37 healthy controls were recruited. Resting EEG was recorded with eyes closed. Participants with $\mathrm{AD} / \mathrm{HD}$ additionally completed EF tasks via the Cambridge Neuropsychological Test Automated Battery. Results: TBR did not differ between groups; however, TBR was positively correlated with inattentive symptoms in AD/HD. Other correlations were found between EEG activity and neuropsychological functions including spatial planning and decision making in the $\mathrm{AD} / \mathrm{HD}$ group. Conclusion: The results do not support the diagnostic value of TBR. Instead, given the heterogeneous features, the results support the prognostic value of EEG in AD/HD.
\end{abstract}

\title{
1 Introduction
}

$\mathrm{AD} / \mathrm{HD}$ is one of the most common neurodevelopmental disorders in the Diagnostic and Statistical Manual of Mental Disorders (4th ed.; DSM-IV; American Psychiatric Association [APA], 1994) among children, and involves pervasive symptoms of inattention, hyperactivity, and impulsivity (APA, 2000). AD/HD is estimated to have a 5.29\% worldwide prevalence (Polanczyk et al., 2007). The disorder often causes functional impairments such as academic, family, and social problems. It can also increase the risk of other psychiatric disorders (Biederman \& Faraone, 2005). As it often begins in early childhood, AD/HD is commonly regarded as a childhood disorder. However, it has been shown that approximately two thirds of those diagnosed in childhood still meet AD/HD criteria in adulthood, leading to long-term negative effects for individuals (Karam et al., 2015).

The electroencephalogram (EEG) allows measurement of brain electrical activity and has been used in $\mathrm{AD} / \mathrm{HD}$ research for decades (for a historical review, see Barry, Clarke, \& Johnstone, 2003) revealing several abnormalities in those with AD/HD compared to healthy controls (Barry \& Clarke, 2009). The most commonly used EEG analysis method is spectral power analysis in which the raw EEG is decomposed into different frequency bands. In childhood, compared to healthy controls, research typically reports that children with AD/HD have increased power in slow wave bands (e.g., absolute theta and delta, and relative theta) and reduced power in fast wave bands (e.g., relative alpha and beta) (Barry et al., 2003; 
Chabot \& Serfontein, 1996). Together, this pattern leads to elevated theta/beta and theta/alpha ratios (Snyder \& Hall, 2006; Snyder et al., 2008). As age increases toward adolescence, the EEG of children with AD/HD shows a typical developmental pattern but the differences remain - that is, increased slow wave and reduced faster wave activity (Barry \& Clarke, 2009; Bresnahan, Anderson, \& Barry, 1999; Clarke, Barry, McCarthy, \& Selikowitz, 2001a). In adulthood, the group differences in fast waves are typically absent, whereas the differences in slower wave activity remain (Barry \& Clarke, 2009; Bresnahan \& Barry, 2002).

Among the observations using EEG, increased theta/beta ratio (TBR) in children with $\mathrm{AD} / \mathrm{HD}$ has been considered to be a robust and reliable group difference. Indeed, the U.S. Food and Drug Administration (2013) approved the utilization of theta/beta ratio to aid assessment of $\mathrm{AD} / \mathrm{HD}$. However, the use of this ratio in aiding diagnosis is debated. $\mathrm{A}$ systematic review revealed that the year of publication had a substantial influence on the reported group difference (Arns et al., 2013). In contrast to earlier findings, recent studies did not find a difference in TBR between groups (Loo et al., 2013; Ogrim, Kropotov, \& Hestad, 2012); a result mainly caused by that the TBR is increasing in healthy control groups (Arns et al., 2013). In addition, age may be a factor that moderates the effect. A study compared TBR between participants with AD/HD and healthy controls in children and adults, and reported that TBR differed in adults but not in children (Loo et al., 2013). However, further studies are needed as the adult controls were the parents of children with AD/HD in this study. Together, these recent findings suggest that the TBR may not be a reliable marker of $\mathrm{AD} / \mathrm{HD}$, and that an age effect exists-the difference may be more obvious in older groups. As a result, concerns about the diagnostic value of TBR are increasing (Arns et al., 2013; Lenartowicz \& Loo, 2014; Saad, Kohn, Clarke, Lagopoulos, \& Hermens, 2015) and more studies are needed to address the issue. Thus, in the current study, we firstly aimed to compare the TBR of AD/HD participants with healthy controls to determine whether TBR differs between groups. Participants were further classified into a child group and an adolescent group to determine whether the difference in TBR changes with age.

The relationship between EEG and behavior/symptoms is another important issue related to AD/HD (e.g., Barry et al., 2009a; Clarke et al., 2011). The most influential EEG model of AD/HD is the hypo-arousal model (Mann et al., 1992; Satterfield \& Cantwell, 1974). The model proposes that abnormalities in EEG indicate central nervous system (CNS) underarousal, which in turn causes behavioral deficiencies. The model can explain the deficit in alpha in $\mathrm{AD} / \mathrm{HD}$ population (Barry, Clarke, Johnstone, \& Rushby, 2008; Barry et al., 2004; 
van Dongen-Boomsma et al., 2010); however, empirical results have shown that differences in beta (Clarke et al., 2013) and TBR (Barry et al., 2009a) in the AD/HD population cannot be explained in terms of arousal. Hence the model, attributing all CNS abnormalities to arousal issues, was too simplistic and the pattern of EEG differences between groups may indicate separate CNS issues (Clarke et al., 2013). Some studies have explored EEG in terms of $\mathrm{AD} / \mathrm{HD}$ symptoms but the results are inconsistent. In a large sample, TBR was positively correlated with inattention symptoms in children, but negatively correlated in adults with $\mathrm{AD} / \mathrm{HD}$ (Loo et al., 2013). This finding is in contrast to a study in which TBR in children was not correlated with inattention but theta was positively correlated with inattention (Ogrim et al., 2012). A correlation between theta and inattention was also reported by Clarke et al. (2011); however, in their study TBR was significantly correlated to hyperactivity/impulsivity symptoms rather than inattention in boys with $\mathrm{AD} / \mathrm{HD}$. Overall, further studies are needed to clarify the behavioral meaning of EEG activity in AD/HD.

New functional insights are emerging from recent studies which examine the behavioral implications of the brain's spontaneous resting activity. The brain's resting state refers to the state where individuals are awake but not performing any task; this is significantly different to a sleep state (Larson-Prior et al., 2011). The nontask-specific “idling" EEG activity, which was previously regarded as relatively meaningless, is increasingly considered as being as informative as neural activity when engaged in a task (Cabral, Kringelbach, \& Deco, 2014; Raichle \& Snyder, 2007). The relationship between resting state and task could potentially be a biomarker of cognitive engagement/load or "work" (Cabral et al., 2014). This is supported by neuroimaging studies which reveal that the relationship between brain activation at rest and during tasks varies across tasks requiring lower- (Cox et al., 2010; Garrett, Kovacevic, McIntosh, \& Grady, 2011; Koyama et al., 2011; Wang et al., 2010) and higher order cognitive process (Reineberg, Andrews-Hanna, Depue, Friedman, \& Banich, 2015). In this context, it has been proposed that behavioral deficits in $\mathrm{AD} / \mathrm{HD}$ may stem from an abnormal brain resting state (Castellanos \& Proal, 2012; SonugaBarke \& Castellanos, 2007).

To date, little research exists on the association between resting EEG and task performance in the $\mathrm{AD} / \mathrm{HD}$ population. In the limited studies that do exist, most have explored the issue in terms of low-level cognitive tasks, for example, theta activity was related to performance in a Continuous Performance Task (Hermens et al., 2005; Shi et al., 2012) and an Oddball task (Hermens et al., 2005). With regard to executive functions (EFs), 
research is rarer and less consistent. EF refers to a set of top-down processes that regulate other, lower level cognitive processes (Diamond, 2013). In several models, some behavioral abnormalities in $\mathrm{AD} / \mathrm{HD}$ are thought to result from executive dysfunctions (e.g., Barkley, 1997; Sergeant, 2005; Sonuga-Barke, 2005). TBR has been related to response inhibition (a component of $\mathrm{EF}$ ), as measured by the Go/Nogo task in children with AD/HD (Loo et al., 2013; van Dongen-Boomsma et al., 2010); however, the relationship was not found when a questionnaire was used to measure inhibition (Ogrim et al., 2012). The relationship between EEG and other components of EF has not been studied. Hence, the second aim of this study was to examine the relationship between EEG bands and multiple components of EF.

Given the above literature review, it was predicted that: TBR in participants with $\mathrm{AD} / \mathrm{HD}$ would differ from that of controls in adolescents whilst this may not be the case in children. Furthermore, based on the lack of directly relevant previous research, we will explore the correlations of EEG outcomes to $\mathrm{AD} / \mathrm{HD}$ symptoms and task performance of $\mathrm{EF}$ in participants with $\mathrm{AD} / \mathrm{HD}$.

\section{Method}

\subsection{Participants}

The AD/HD participants were recruited between June 2014 and June 2015 at Peking University Sixth Hospital. They were assessed by experienced psychiatrists. All participants met the following inclusion criteria: (a) screened by the Clinical Diagnostic Interviewing Scales (Barkley, 1998), a structured clinical interview based on the DSM-IV; (b) no history of head trauma with loss of consciousness; (c) no history of neurological illness or other severe disease; (d) no history of psychiatric disorders described in the DSM-IV; (e) naive to any pharmacological treatment; and (f) an IQ higher than 80 on the Wechsler Intelligence Scale III for children.

Fifty-three children (43 male, age range: 8 -15 years, $\mathrm{M}=11.24$ years, $\mathrm{SD}=2.15$ ) with $\mathrm{AD} / \mathrm{HD}$ were selected from $101 \mathrm{AD} / \mathrm{HD}$ cases in this study as they were aged from 8 to 15 years and had full neuropsychological testing reports. Thirty-two children with AD/HD were diagnosed with the predominantly inattentive type (ADHD-I) and 21 with the combined type (ADHD-C). Two age groups were formed: a child group ranging from 8 to 12 years and an adolescent group from 12 to 15 years. For children with AD/HD, 40 children were entered into the group analysis after balancing age and sex compared to controls. There were 24 participants in the child group (13 ADHD-I, 11 ADHD-C, 18 male, $\mathrm{M}=10.20$ years, $\mathrm{SD}=$ 
0.70) and 16 (9 ADHD-I, 7 ADHD-C, 12 male, $\mathrm{M}=13.45$ years, $\mathrm{SD}=0.60$ ) in the adolescent group. In the correlational analysis, all children with AD/HD were considered.

Thirty-seven healthy controls (27 male, age range: $8-15$ years, $\mathrm{M}=12.09$ years, $\mathrm{SD}=$ 1.68) participated in this study. There were 18 participants in the child group (14 male, $\mathrm{M}=$ 10.50 years, $\mathrm{SD}=0.77$ ) and 19 participants in the adolescent group (14 male, $\mathrm{M}=13.60$ years, $\mathrm{SD}=0.38$ ). Healthy controls were recruited from the hospital and local schools and were screened by the same psychiatrists with the same inclusion criteria applied but they did not meet the diagnostic criteria of $\mathrm{AD} / \mathrm{HD}$; they met less than four $\mathrm{DSM}-\mathrm{IV}$ Inattention criteria and four DSM-IV Hyperactivity/Impulsivity criteria.

\subsection{Procedure}

Ethics approval was obtained from the Ethics Committee of Peking University Health Science Center and the University of Wollongong Human Research Ethics Committee. Informed consent was obtained from the parent or guardian of each participant prior to accessing any record or testing.

Participants were required to complete the testing protocol in 1 day. In the morning, participants were in a patient room accompanied by a psychiatrist. They completed psychometric assessments and the Cambridge Neuropsychological Test Automated Battery (CANTAB). These processes lasted about $2 \mathrm{hr}$. In the afternoon, resting EEG was recorded in a room which was free from distraction, with participants seated on a comfortable chair with dimmed lighting. This process lasted about $45 \mathrm{~min}$.

\subsection{CANTAB}

CANTAB is a commercial computerized neuropsychological battery consisting of a wide range of cognitive tasks. Based on the research purpose of this study, five tasks related to $\mathrm{EFs}$ and deficiencies in $\mathrm{AD} / \mathrm{HD}$ were used; see below. Each task contained several outcomes with the main measures reported here.

The Stockings of Cambridge (SOC) task measures spatial planning. Three colored balls are displayed in a spatial pattern and participants are required to move the other set of balls to repeat the pattern. Outcomes reported are (a) problems solved in minimum moves (PSMM), (b) mean initial thinking time (ITT): the mean time taken before moving the ball, and (c) mean subsequent thinking time (STT): the mean time taken after the initial move. The task lasts for about $10 \mathrm{~min}$. 
The Intra-Extra Dimensional Set Shift (IED) task measures cognitive flexibility. The task is similar to the Wisconsin Card Sorting test, and requires participants to learn a rule and then to shift from the well-learned rule to a new rule. Outcomes reported is stages completed (SC): the total number of successfully completed stages. The task lasts for about $7 \mathrm{~min}$.

The Stop Signal task (SST) measures response inhibition. The task firstly builds up a tendency for participants to make an A or B response, and on a small percentage (e.g., 25\%) of trials, participants are required to inhibit the activated response after hearing a tone (which is presented at various times poststimulus presentation; for example, $50 \mathrm{~ms}, 150 \mathrm{~ms}$, and 300 $\mathrm{ms}$ ). The primary outcome is stop signal reaction time (SSRT) which is an estimate of the time taken to inhibit the response. The task lasts for about $20 \mathrm{~min}$.

The Spatial Working Memory (SWM) task measures the visuospatial ability of working memory. Tokens are spatially hidden in the task and participants need to find the token by trying different spatial locations. Outcomes include (a) between errors (BE): defined as revisiting the place in which a token has already been found, and (b) within errors (WE): defined as revisiting the place in which no token has already been found.

The Cambridge Gambling Task (CGT) measures decision making. Participants are presented with 10 boxes which are red and blue, and are required to guess whether a yellow token is hidden in a red box or a blue box. Outcomes are (a) delay aversion (DA): participants score higher if they are unable or unwilling to wait, and (b) betting proportion (BP): the overall proportion of bets across trials.

The SOC, IED, SST, and SWM tasks were used to measure differing components of EF. CGT was included as it measures decision making as a higher order function based on EF (Diamond, 2013). Full illustrations and demonstrations for each task are available in a review article (Chamberlain et al., 2011).

\subsection{EEG Recording and Pre-Processing}

Ten minutes of EEG was recorded in an eyes-closed resting condition. The recording was paused if the participant showed signs of fatigue or restlessness. The EEG was acquired using a 128-channel system (HydroCel Geodesic Sensor Net, Electrical Geodesics, Inc., Eugene, OR). The impedance of all electrodes was less than $50 \mathrm{k} \Omega$. All electrodes were physically referenced to $\mathrm{Cz}$ (fixed by the EGI system). The EEG was amplified with a band 
pass of 0.01 to $200 \mathrm{~Hz}$, which was digitized online at a sampling rate of 1,000 Hz. The EGI data were converted to allow analysis using EEGLAB and Neuroscan software Version 4.3.

Nineteen channels were selected based on the international 10-20 system. All channels were offline re-referenced to linked ears, and resampled at $256 \mathrm{~Hz}$, filtered by a band-pass filter from $1 \mathrm{~Hz}$ to $70 \mathrm{~Hz}$ and a $50-\mathrm{Hz}$ notch filter. Visual inspection was used to identify and exclude sections of EEG trace containing gross artifacts. The Independent Component Analysis function in EEGLAB (Delorme \& Makeig, 2004) identified components related to eye and muscle movements and there were excluded; this is a semiautomatic process aided by a tool box in EEGLAB, ADJUST (Mognon, Jovicich, Bruzzone, \& Buiatti, 2011). Then, in line with earlier resting EEG studies, the first 3 min were extracted from the artifact-free EEG data and were segmented into 4-s epochs. These epochs were Fourier transformed using a Hamming window. Summed EEG band power was calculated for four frequency bands: delta $(1.5-3.5 \mathrm{~Hz})$, theta $(3.5-7.5 \mathrm{~Hz})$, alpha $(7.5-12.5 \mathrm{~Hz})$, and beta $(12.5-$ $25 \mathrm{~Hz}$ ). The total power and relative power of four bands were used in the statistical analysis as these two indices are reliable to characterize EEG features in participants with $\mathrm{AD} / \mathrm{HD}$ (Clarke et al., 2011). Relative power was calculated by dividing absolute power in each frequency band by the total of bands.

\subsection{Statistical Analysis}

ANOVAs with between-subjects factors of Age (child, adolescent) and Group (AD/HD, control) and within-subjects topographic factors of Lateral (left, midline, right) and Sagittal (frontal, central, posterior) were conducted for the theta/beta ratio, and separately for each frequency band in relative power. Topographic effects were examined using an established method (Clarke et al., 2001a). All electrodes were divided into nine regions: left frontal (F3, F7), midline frontal (Fz), right frontal (F4, F8), left central (T3, C3), midline central $(\mathrm{Cz})$, right central $(\mathrm{T} 4, \mathrm{C} 4)$, left posterior $(\mathrm{T} 5, \mathrm{P} 3, \mathrm{O} 1)$, midline posterior $(\mathrm{Pz})$, and right posterior (T6, P4, O2). The EEG data for each region were calculated by averaging all electrode(s) within the area. Planned contrasts were examined within the Sagittal and Lateral factors. Within the Sagittal factor, planned contrasts compared the frontal (F) and posterior regions $(\mathrm{P})$, and the central region $(\mathrm{C})$ with the mean of the frontal and posterior regions $(\mathrm{F} / \mathrm{P})$. Within the Lateral factor, the contrasts compared the left hemisphere (L) with the right $(\mathrm{R})$, and the midline region $(\mathrm{M})$ with the mean of the hemispheres $(\mathrm{L} / \mathrm{R})$. The contrasts are 
planned, and there are no more of them than the degrees of freedom for the effect, so no Bonferroni-type adjustment to $\alpha$ is required (Tabachnick \& Fidell, 2007).

To examine the influence of age and IQ on behavioral performance, partial correlations separately examined the relationships between EEG band power/percentage and scores from the AD/HD Rating Scale (ADHD-RS) and task performance measures from the CANTAB with age and IQ co-varied. Only the Sagittal factor was entered into analyses to reduce the number of correlations and the risk of Type 1 error from testing multiple correlations. The significance level for the correlations was adjusted to a more conservative .01; meanwhile, the significance close to .01 is also reported as this is a preliminary study $(p \leq .015)$.

\section{Results}

Demographic information for the groups is shown in Table 1. The children with $\mathrm{AD} / \mathrm{HD}$ and controls did not differ significantly in age. A main effect of Group was significant for IQ, and indicated that participants with $\mathrm{AD} / \mathrm{HD}(\mathrm{M}=103.98, \mathrm{SD}=11.36)$ had a lower IQ than controls $(\mathrm{M}=123.92, \mathrm{SD}=8.98), F=70.44, p<.001, \eta_{p}^{2}=0.492$.

Table 1. Demographic Information for the AD/HD and Control Groups.

\begin{tabular}{|c|c|c|c|c|}
\hline & Child AD/HD & $\begin{array}{c}\text { Adolescent } \\
\text { AD/HD }\end{array}$ & Child control & $\begin{array}{l}\text { Adolescent } \\
\text { control }\end{array}$ \\
\hline Gender & $8 \mathrm{~F} ; 16 \mathrm{M}$ & $4 \mathrm{~F} ; 12 \mathrm{M}$ & $4 \mathrm{~F} ; 14 \mathrm{M}$ & $5 \mathrm{~F} ; 14 \mathrm{M}$ \\
\hline Mean age in years & $10.18(0.70)$ & $13.45(0.60)$ & $10.49(0.77)$ & $13.59(0.38)$ \\
\hline WISC Full Scale IQ & $104.42(12.05)$ & $103.31(10.57)$ & $121.89(9.44)$ & $125.84(8.31)$ \\
\hline AD/HD combined type & 11 & 7 & - & - \\
\hline $\mathrm{AD} / \mathrm{HD}$ inattentive type & 13 & 9 & - & - \\
\hline
\end{tabular}

Note. Numbers in brackets represent standard deviation. WISC $=$ Wechsler Intelligence Scale III for children.

\subsection{Delta}

Fig. 1 and Fig. 2 display brain maps for the two groups for relative power of each EEG band, TBR and total power. A Sagittal main effect and planned contrasts (linear: $F=$ 132.178, $p<.001,=0.644$; quadratic: $\left.F=5.459, p=.022, \eta_{p}^{2}=0.070\right)$ indicated that delta was maximal in the fronto-central region. Main effects of Group $\left(F=7.501, p=.008, \eta_{p}^{2}=\right.$ $0.093)$ and Age $\left(F=13.776, p<.001, \eta_{p}^{2}=0.159\right)$ indicated that children with AD/HD had globally increased delta power compared to controls, and that adolescents showed decreased 
delta power compared to children. An Age $\times$ Sagittal interaction (quadratic, $F=3.991, p$ $\left.=.049, \eta_{p}^{2}=0.058\right)$ revealed that the difference between $\mathrm{C}$ and $\mathrm{F} / \mathrm{P}$ was larger in adolescents than children, indicating that the reduction with age was more obvious in frontal and posterior regions.

AD/HD
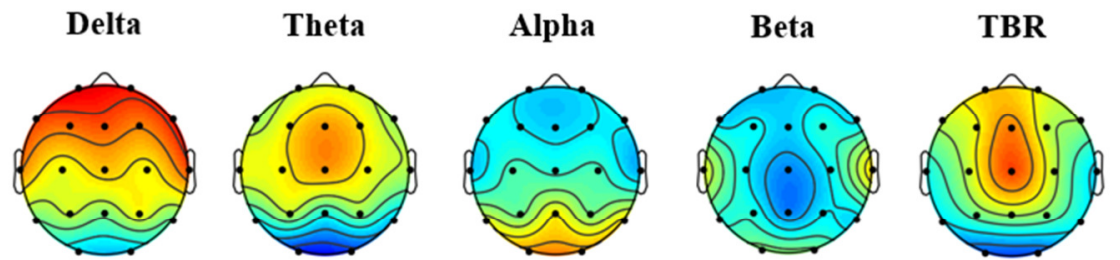

Total Power

Control
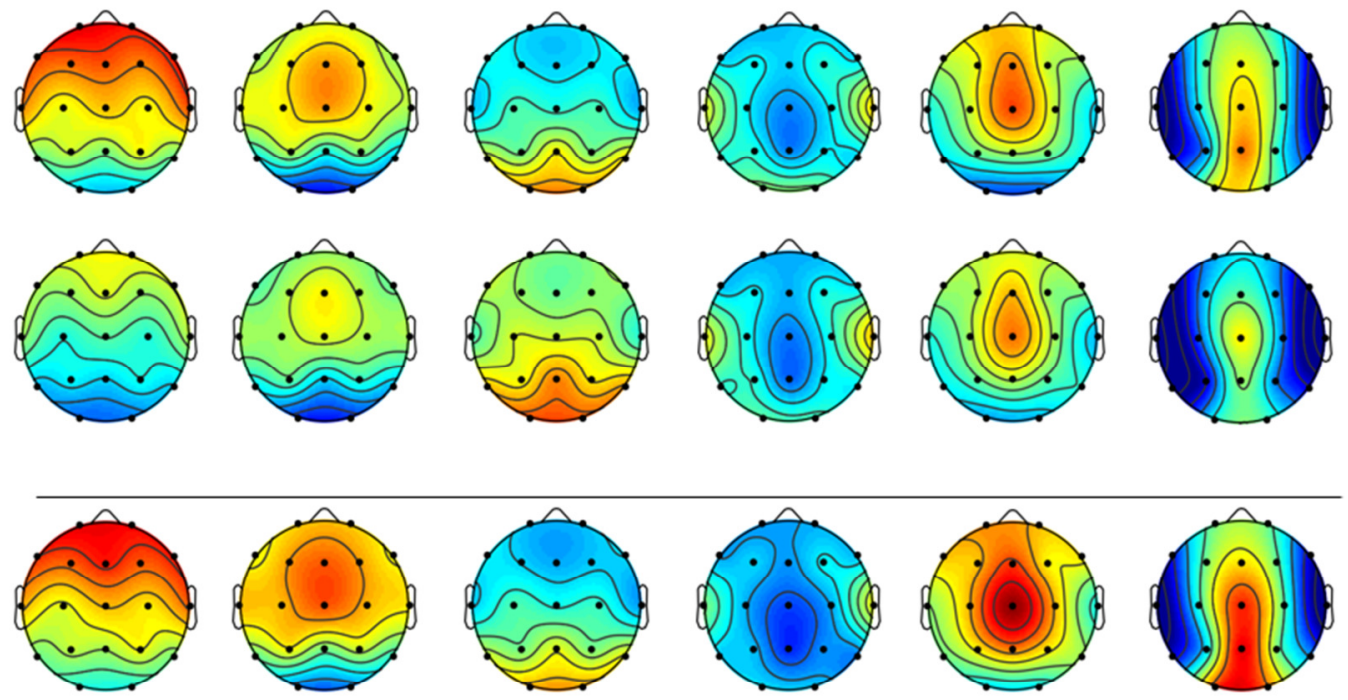

Children
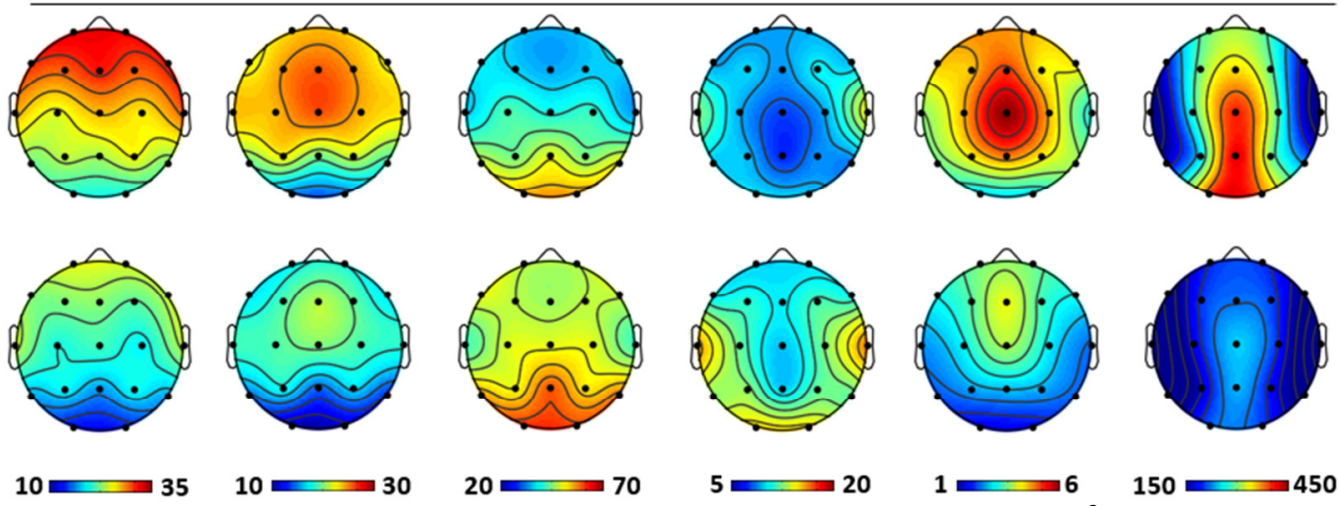

Fig. 1. Topographic maps for relative power $(\%), T B R$, and total power $\left(\mu \mathrm{V}^{2}\right)$ for each level of Group (top panel), and each level of Age (bottom panel). Note. TBR = theta/beta ratio.

\subsection{Theta}

Laterally, theta was maximal in the midline region (quadratic: $F=77.421, p<.001$, $\eta_{p}^{2}=0.515$ ). The Sagittal main effect and planned contrasts (linear: $F=82.741, p<.001$, $\eta_{p}^{2}=0.531$; quadratic: $\left.F=187.985, p<.001, \eta_{p}^{2}=0.720\right)$ indicated a fronto-central distribution. A main effect of Age $\left(F=12.171, p=.001, \eta_{p}^{2}=0.143\right)$ indicated that theta decreased with increasing age. An Age $\times \operatorname{Group}\left(F=4.354, p=.040, \eta_{p}^{2}=0.056\right)$ interaction revealed that theta decreased more with age in the $\mathrm{AD} / \mathrm{HD}$ group compared to controls. $\mathrm{A}$ Group $\times$ Age $\times$ Sagittal (linear) interaction $\left(F=4.305, p=.042, \eta_{p}^{2}=0.056\right)$ indicated the Age $\times$ Group interaction was less marked at frontal than posterior regions. This was mainly driven by the elevated theta power in frontal regions in the AD/HD group. 


\subsection{Alpha}
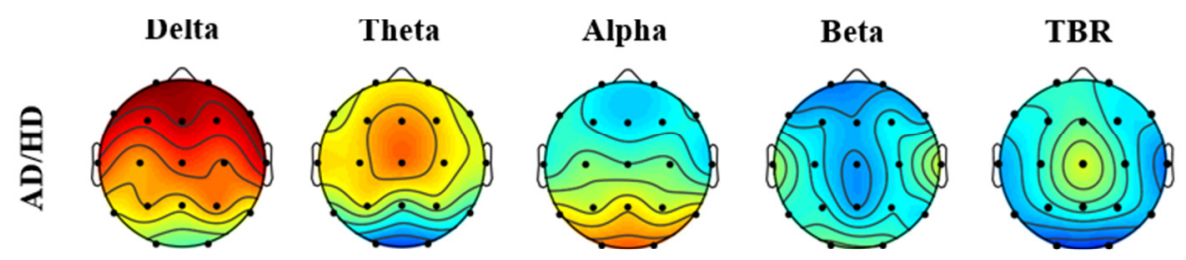

Total Power

Children
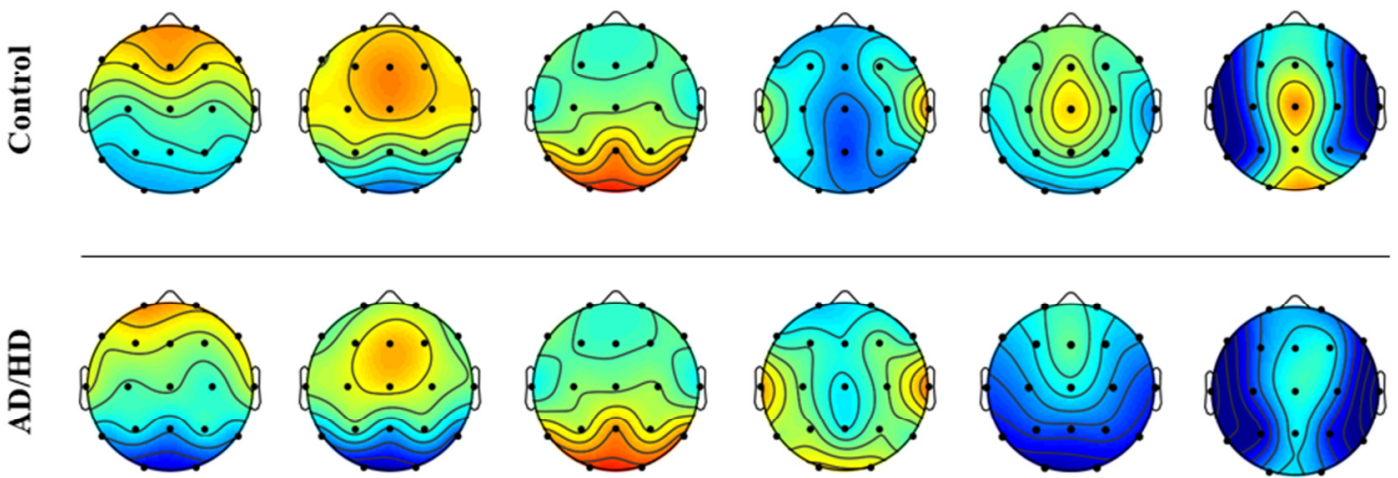

Adolescents
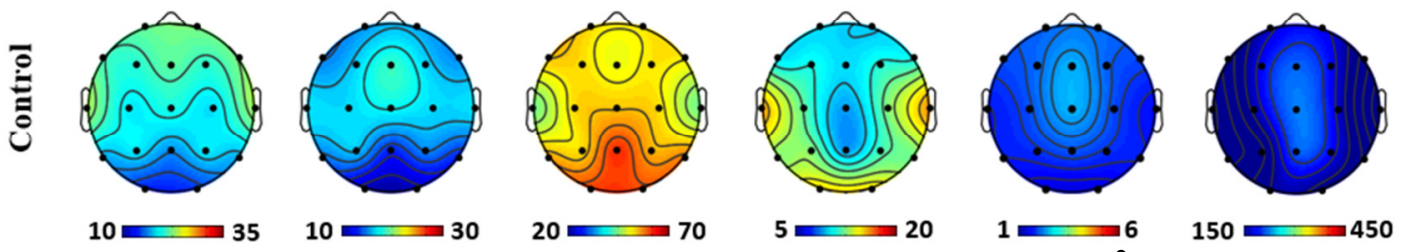

Fig. 2. Topographic maps for relative power $(\%), T B R$, and total power $\left(\mu V^{2}\right)$, for each level of Age and Group separately. Note. TBR $=$ theta/beta ratio.

The Sagittal effect and contrasts (linear: $F=126.853, p<.001, \eta_{p}^{2}=0.635$; quadratic: $\left.F=109.483, p<.001, \eta_{p}^{2}=0.600\right)$ revealed that alpha was maximal in the posterior region. Main effects of Group $\left(F=4.384, p=.040, \eta_{p}^{2}=0.057\right)$ and Age $\left(F=9.204, p=.003, \eta_{p}^{2}=\right.$ 0.112 ) indicated that children with $\mathrm{AD} / \mathrm{HD}$ had globally decreased alpha, and that adolescents had increased alpha compared to children. An Age $\times$ Sagittal interaction (linear, $F=4.480, p$ $=.038, \eta_{p}^{2}=0.058$ ) showed that the difference between $\mathrm{F}$ and Pwas larger in adolescents, an effect mainly driven by the larger increase in posterior regions in adolescents.

\subsection{Beta}

Laterally, beta was maximal in the hemispheres compared to the midline (quadratic, $F$ $\left.=192.912 . p<.001, \eta_{p}^{2}=0.725\right)$. There was a central maximal distribution along the Sagittal dimension (quadratic, $\left.F=32.260, p<0.001, \eta_{p}^{2}=0.306\right)$. An Age main effect $(F=8.250, p$ 
$\left.=.005, \eta_{p}^{2}=0.102\right)$ showed that beta was increased in adolescents compared to children. An Age $\times$ Sagittal interaction (linear, $F=10.850, p=.002, \eta_{p}^{2}=0.129$ ) revealed that the difference between $\mathrm{F}$ and $\mathrm{P}$ was increased in the adolescent group with a larger increase in the posterior region. No Group effect or any interactions with Group were found.

\subsection{TBR}

TBR had a left-midline distribution along the Lateral dimension (linear: $F=5.519, p$ $=.022, \eta_{p}^{2}=0.070$; quadratic: $\left.F=141.927, p<.001, \eta_{p}^{2}=0.660\right)$. The Sagittal analysis (linear: $F=24.426, p<.001, \eta_{p}^{2}=0.251$; quadratic, $F=8.752, p=.004, \eta_{p}^{2}=0.107$ ) indicated that TBR was maximal in the fronto-central region. An Age main effect $(F=$ 12.219, $\left.p=.001, \eta_{p}^{2}=0.143\right)$ indicated that TBR decreased with age. An Age $\times$ Lateral interaction (quadratic, $F=7.064, p=.010, \eta_{p}^{2}=0.088$ ) indicated that the difference between $\mathrm{M}$ and $\mathrm{L} / \mathrm{R}$ was reduced in adolescents, which is mainly caused by the larger reduction in the midline region. No Group effect or interaction with Group was found.

\subsection{Total Power}

A Sagittal main effect and planned contrasts (linear: $F=5.846, p=.018, \eta_{p}^{2}=0.075$; quadratic: $\left.F=13.176, p=.001, \eta_{p}^{2}=0.155\right)$ indicated that total power was maximal in central-posterior regions. Total power was maximal in the middle line (quadratic, $F=84.153$, $p<.000, \eta_{p}^{2}=0.539$. An Age main effect $\left(F=9.049, p=.004, \eta_{p}^{2}=0.112\right)$ indicated that total power was decreased in adolescents compared to children. An Age $\times$ Sagittal interaction (linear, $F=7.709, p=.007, \eta_{p}^{2}=0.097$ ) showed that the difference between $\mathrm{F}$ and $\mathrm{P}$ was larger in adolescents, the effect mainly caused by the larger reduction in the posterior region in adolescents. No Group effect or interaction with Group was found.

\subsection{Behavioral Correlates}

Partial correlations were conducted among participants with AD/HD to analyze the relationship between EEG and the behavioral measures with age and IQ as covariates. The correlations are shown in Table 2. 
Table 2. Correlations Between EEG and Behavioral Measures.

\begin{tabular}{lllll}
\hline & Theta & Beta & TBR & Total power \\
\hline Inattention & - & $\mathrm{F}, r=-.373$ & $\mathrm{~F}, r=.411$ & - \\
ADHD-RS & & & & - \\
SOC & $\mathrm{C}, r=.330$ & - & - & \\
STT & $\mathrm{P}, r=.398$ & & & $\mathrm{~F}, r=.360$ \\
CGT & - & - & - & $\mathrm{P}, r=.347$ \\
BP & & & & \\
\hline
\end{tabular}

Note. EEG indices (Delta and Alpha) and behavioral measures (Hyperactivity-Impulsivity, IED, SST, and SWM) were not listed as no significant correlations were found for them. Regional abbreviation: $\mathrm{F}$, frontal; $\mathrm{C}$, central; $\mathrm{P}$, posterior. $\mathrm{EEG}=$ electroencephalogram; TBR $=$ theta/beta ratio; $\mathrm{ADHD}-\mathrm{RS}=\mathrm{AD} / \mathrm{HD}$ Rating Scale; $\mathrm{SOC}=$ Stockings of Cambridge; $\mathrm{STT}=$ subsequent thinking time; CGT = Cambridge Gambling Task; $\mathrm{BP}=$ betting proportion; IED = Intra-Extra Dimensional Set Shift; SST = Stop Signal task; SWM = Spatial Working Memory.

With regard to symptoms, the inattention score measured by the ADHD-RS was significantly correlated with frontal beta power $(r=.304, p=.027)$, and frontal TBR ratio $(r$ $=.379, p=.005)$. No EEG outcome was related to hyperactivity and impulsivity measured by the ADHD-RS.

For EF, central $(r=.330, p=.016)$ and posterior $(r=.398, p=.003)$ theta power was positively correlated with STT in spatial planning (SOC); thus, increased frontal/posterior theta and posterior TBR are related to a slower response. For decision making (CGT), frontal $(r=.360, p=.008)$ and posterior $(r=.347, p=.011)$ total power were positively related to the amount of betting behavior, indicating an increased total power accompanied greater risk taking behavior. No relationship was found with the shifting, response inhibition, and working memory tasks.

\section{Discussion}

The diagnostic value of TBR has recently been questioned in the literature. Research shows that the difference in TBR between participants with AD/HD and healthy controls has decreased in recent years, and that age may affect the group difference. To investigate this further, the current study compared EEG spectral power, including TBR, between participants with $\mathrm{AD} / \mathrm{HD}$ and healthy controls in child and adolescent groups. Moreover, we explored the relationship between resting EEG and a range of neuropsychological functions to further understand the behavioral relevance of brain resting activities. 


\subsection{Relative and Total Power}

Previous studies report that, as age increases, higher frequency brain activity decreases and lower frequency activity increases, with decreased total power (Barry \& Clarke, 2009). Consistent with previous results, the current study found reduced delta, theta, and total power, and increased alpha and beta in adolescents compared to children. With regard to the comparison between groups, participants with $\mathrm{AD} / \mathrm{HD}$ showed more delta and less alpha, which is in line with past studies indicating that $\mathrm{AD} / \mathrm{HD}$ is accompanied by increased slow wave activity and reduced faster activity (Barry \& Clarke, 2009; Barry et al., 2003). The interaction between Group and Age for theta, which decreased slower in the $\mathrm{AD} / \mathrm{HD}$ group than control group with increasing age, may indicate a developmental deviation. The result is in line with developmental EEG studies in which theta activity was still abnormal in adults with $\mathrm{AD} / \mathrm{HD}$ whereas abnormalities in other bands approached normal levels (Barry \& Clarke, 2009).

Frontal beta and frontal TBR were associated with the inattention score measure of the ADHD-RS. These results are consistent with recent reports on adults with AD/HD. Roh et al. (2015) and Roh, Park, Shim, and Lee (2016) reported that inattention issues were linked to theta, beta, and also gamma activity. The multiple relationships between elements of the EEG and $\mathrm{AD} / \mathrm{HD}$ symptoms fits well with comprehensive AD/HD models, such as CognitiveEnergetic Model (Sergeant, 2005) and multiple pathways model (Sonuga-Barke, 2005). These models commonly emphasize that different resources contribute to symptoms.

The relationship of EEG to EF task performance was also examined in the AD/HD group, with correlations found with spatial planning and decision making. These results are in line with recent developments in cognitive neuroscience that examine behavioral relationships with brain resting activity. The interest stems from the perspective that brain resting activity has consistent networks across individuals and the networks are varied when relevant tasks are presented (Cabral et al., 2014; Raichle \& Snyder, 2007). The point was subsequently supported by imaging studies in which the resting networks were correlated with some attention and EF tasks (Cabral et al., 2014; Reineberg et al., 2015). Studies further classified the brain resting networks as "task-negative" or "task-positive." The former refers to networks that are activated in resting state and will be decreased when tasks emerge, and the latter refer to other networks that are activated during rest but will be increased when tasks emerge (Cabral et al., 2014). In the present study, the resting state was measured by 
EEG, and correlations were found in AD/HD participants. To summarize (a) central and posterior relative theta was positively related to planning time; (b) frontal and posterior total absolute power were positively related to betting in decision making.

\subsection{TBR}

In the current study, TBR decreased with increasing age across groups, which is consistent with the developmental pattern revealed by other studies (Barry \& Clarke, 2009). TBR did not differ between ADHD and control groups in either children or adolescents. This result contradicts early studies in which a significantly increased TBR was consistently observed in participants with AD/HD compared to healthy controls. In 2006, a meta-analysis reported that the effect size of the TBR group difference was 3.08 (Snyder \& Hall, 2006). However, our results support a trend exposed by Arns et al. (2013) in which the effect size of the group difference was negatively related to the year of publication. This reduction was more obvious for the studies published after 2008. Consequently, two recent studies did not report a significant TBR difference between groups (Loo et al., 2013; Ogrim et al., 2012). In the current study, the lack of a group difference was replicated within two age-ranges (children: 8-12 years, adolescents: 12-15 years) in a Chinese population. Close inspection shows that the value of TBR is elevated in our controls whereas it remains similar in AD/HD group compared to previous studies, which supports the view that it is a change in controls over the years that has reduced the difference between groups (Arns et al., 2013). Arns et al. (2013) speculated that changes in living habits, such as decreasing sleep duration, may be responsible for this effect. As no data were recorded for sleep, this assumption cannot be examined in the current study.

TBR was positively correlated to inattention symptoms. This result matches the studies that support the relationship between TBR and AD/HD symptoms in children (e.g., Loo et al., 2013). The result is also in parallel with findings in the normal population. Putman, van Peer, Maimari, and van der Werff (2010) reported that higher frontal TBR was related to poorer attentional control and response inhibition performance. Although we did not find a relationship between TBR and response inhibition, this may be because of the difference in the task paradigms. A stop-signal task was used to measure response inhibition in the current study, while an emotional Go/Nogo task was used in the Putman study. However, the correlation between TBR and inattention is contrary to Ogrim et al. (2012) in which the relationship was not found, which may be attributed to methodological differences. 
The EEG was recorded under eyes-open condition and different frequency bands were used in our study compared to Ogrim et al. (2012).

\subsection{Implications and Future Studies}

Along with recent findings, our results do not support the diagnostic value of TBR among children with AD/HD (Arns et al., 2013; Lenartowicz \& Loo, 2014). Given the age range of the present study (8-15 years), future studies may extend the findings to the later stage of adolescent. AD/HD symptoms are usually varied in children as compared to adults, with implications for EEG. The increasing ratio in controls over time is suggested to reduce the group difference (Arns et al., 2013). Further studies may examine whether changes of lifestyle and habits in healthy control children is leading to the increasing TBR, as suggested by Arns et al. (2013). It should be noted that no healthy controls in this study had substantial inattention symptoms (they met less than four DSM-IV inattention criteria), which also raises a question whether TBR has the same behavioral meaning in AD/HD and healthy populations, given the result that TBR was positively related to inattention symptom in $\mathrm{AD} / \mathrm{HD}$. In addition, the heterogeneous nature of $\mathrm{TBR}$ in the $\mathrm{AD} / \mathrm{HD}$ population may contribute to the lack of difference in TBR (Arns et al., 2013). According to a cluster analysis, $35 \%$ of the AD/HD population was characterized as having a higher TBR and the others showed different EEG deficit patterns (Clarke et al., 2011).

The heterogeneous feature was not only found in EEG studies but also in neuropsychological research (Nigg et al., 2005). The neuropsychological deficits in AD/HD were initially explained as a core inhibitory deficit (Barkley, 1997). However, researchers subsequently found that not all participants with AD/HD showed inhibitory deficits (Willcutt, Doyle, Nigg, Faraone, \& Pennington, 2005) and a neuropsychological AD/HD subtype was suggested (Nigg et al., 2005). Given the neuropsychological correlations found in the current study, the heterogeneous EEG may be associated with the heterogeneous neuropsychological functions. In other words, individual differences in EEG may indicate differences in neuropsychological functions. It should be noted that spatial planning is regarded as a component of EF in CANTAB; however, planning ability, as decision making in the 3component EF model, is considered a higher level cognitive function based on the three EF components (inhibition, shifting and working memory; Diamond, 2013; Miyake et al., 2000). In this study, EEG outcomes (theta power and total power) are associated with spatial planning and betting in decision making but none of the EEG outcomes are related to 
response inhibition, shifting and working memory. Following the EF model, the resting EEG measures are indicative of two higher cognitive functions (planning and decision making) rather than $\mathrm{EF}$ in $\mathrm{AD} / \mathrm{HD}$.

Practically, the behavioral correlations with EEG support the prognostic value of EEG in $\mathrm{AD} / \mathrm{HD}$. As EEG is doubtful in diagnosing $\mathrm{AD} / \mathrm{HD}$, a prognostic purpose is suggested for using EEG in AD/HD (Arns et al., 2013; Lenartowicz \& Loo, 2014). The suggestion stems from the findings that EEG can predict the efficacy of stimulant treatment in patients with AD/HD (Arns, 2012; Clarke, Barry, McCarthy, \& Selikowitz, 2002a). Based on current results, EEG components may be prognostically used as biomarkers of neuropsychological functions. Theoretically, the correlations also support a neurobiological hypothesis in AD/HD (Castellanos \& Proal, 2012; Sonuga-Barke \& Castellanos, 2007). The hypothesis suggests the behavioral deficits in AD/HD are neurobiologically rooted in impaired spontaneous activities. Recently, Hsu, Benikos, and Sonuga-Barke (2015) and Hsu, Broyd, Helps, Benikos, and Sonuga-Barke (2013) reported that the resting state was linked to waiting-related behaviors in an $\mathrm{AD} / \mathrm{HD}$ sample. Here, we have extended this to look at the relationship between resting state and EF and higher order cognitive functions. However, the current study used a different definition of resting state from that of Hsu and colleagues. Here, the resting state was defined in terms of traditional EEG spectrum bands. These bands have been shown to be activated in a similar pattern across individuals (Chen, Feng, Zhao, Yin, \& Wang, 2008) and to relate to resting networks measured by functional magnetic resonance imaging (Mantini, Perrucci, Del Gratta, Romani, \& Corbetta, 2007). In comparison, Hsu et al. (2015) and Hsu et al. (2013) directly adopted a similar methodology to imaging studies and operationalized the resting state by measuring the EEG oscillation in a narrow band of 'very low frequency' (below 0.2 Hz). Further studies may explore differences between these two 'resting state' measures in an $\mathrm{AD} / \mathrm{HD}$ sample.

\subsection{Limitations}

A limitation of this study is that IQ in controls is higher than the AD/HD group and the typical population. Here, we followed a standard methodology that shows little influence of IQ in EEG group analyses (Barry et al., 2009; Clarke et al., 2006), and it would have been preferable to avoid such a difference. Also, given the preliminary nature of the behavioral correlational analysis in $\mathrm{AD} / \mathrm{HD}$, the neuropsychological functions were not recorded in the control groups and more information may have been obtained by comparing the behavioral 
correlations in the different groups. In addition, although CANTAB is a valid tool to measure a wide range of neuropsychological functions (Chamberlain et al., 2011), with regard to the complicated features of EF and higher order cognitive functions, measuring the functions by only one task in CANTAB leaves open the issue of task impurity (Diamond, 2013; Miyake et al., 2000). For example, the individual difference in response time on spatial planning may be caused by the variance on the lower order reaction process rather than on the planning per se. Factor analysis is accepted as a better way to extract the purer components (Miyake et al., 2000). Future studies may further explore the behavioral relationships of EEG by using factor analysis with a number of different tasks.

\section{Conclusion}

With recent debates on the diagnostic value of TBR in AD/HD, the aim of the current study was to compare TBR between AD/HD and control groups of children and adolescents. In line with recent studies, we did not find a group difference on TBR across the age range, which does not support the diagnostic value of TBR in AD/HD. However, inspired by recent findings in cognitive neuroscience, the current study explored the relationship between EEG spectral power and a range of neurophysiological tasks in participants with $\mathrm{AD} / \mathrm{HD}$. With the significant correlations, the results support the prognostic value of resting EEG which may be biomarkers of neuropsychological functions. 


\section{References}

American Psychiatric Association. (1994). Diagnostic and statistical manual of mental disorders (4th ed.). Washington, DC: Author.

American Psychiatric Association. (2000). Diagnostic and statistical manual of mental disorders (4th ed., text rev.). Washington, DC: Author.

Arns, M. (2012). EEG-based personalized medicine in ADHD: individual alpha peak frequency as an endophenotype associated with nonresponse. Journal of Neurotherapy, 16(2), 123-141.

Arns, M., Conners, C. K., \& Kraemer, H. C. (2013). A decade of EEG Theta/Beta ratio research in ADHD: A meta-analysis. Journal of Attention Disorders, 17, 374-383.

Barkley, R. A. (1997). Behavioral inhibition, sustained attention, and executive functions: Constructing a unifying theory of ADHD. Psychological Bulletin, 121, 65-94.

Barkley, R. A. (1998). Attention-deficit hyperactivity disorder : A handbook for diagnosis and treatment (2nd ed.). New York, NY: Guilford Press.

Barry, R. J., \& Clarke, A. R. (2009). Spontaneous EEG oscillations in children, adolescents, and adults. Journal of Psychophysiology, 23(4), 157-173.

Barry, R. J., Clarke, A. R., \& Johnstone, S. J. (2003). A review of electrophysiology in attention-deficit/hyperactivity disorder: I. Qualitative and quantitative electroencephalography. Clinical Neurophysiology, 114, 171-183.

Barry, R. J., Clarke, A. R., Johnstone, S. J., McCarthy, R., \& Selikowitz, M. (2009). Electroencephalogram theta/beta ratio and arousal in attention-deficit/hyperactivity disorder: Evidence of independent processes. Biological Psychiatry, 66, 398-401.

Barry, R. J., Clarke, A. R., Johnstone, S. J., \& Rushby, J. A. (2008). Timing of caffeine's impact on autonomic and central nervous system measures: Clarification of arousal effects. Biological Psychology, 77, 304-316.

Barry, R. J., Clarke, A. R., McCarthy, R., Selikowitz, M., Rushby, J. A., \& Ploskova, E. (2004). EEG differences in children as a function of resting-state arousal level. Clinical Neurophysiology, 115, 402-408.

Biederman, J., \& Faraone, S. V. (2005). Attention-deficit hyperactivity disorder. The Lancet, $366,237-248$.

Bresnahan, S. M., Anderson, J. W., \& Barry, R. J. (1999). Age-related changes in quantitative EEG in attention-deficit/hyperactivity disorder. Biological Psychiatry, 46, 1690-1697.

Bresnahan, S. M., \& Barry, R. J. (2002). Specificity of quantitative EEG analysis in adults with attention deficit hyperactivity disorder. Psychiatry Research, 112, 133-144.

Cabral, J., Kringelbach, M. L., \& Deco, G. (2014). Exploring the network dynamics underlying brain activity during rest. Progress in Neurobiology, 114, 102-131.

Castellanos, F. X., \& Proal, E. (2012). Large-scale brain systems in ADHD: Beyond the prefrontal-striatal model. Trends in Cognitive Sciences, 16, 17-26.

Chabot, R. J., \& Serfontein, G. (1996). Quantitative electroencephalographic profiles of children with attention deficit disorder. Biological Psychiatry, 40, 951-963.

Chamberlain, S. R., Robbins, T. W., Winder-Rhodes, S., Muller, U., Sahakian, B. J., Blackwell, A. D., \& Barnett, J. H. (2011). Translational approaches to frontostriatal dysfunction in attention-deficit/hyperactivity disorder using a Computerized Neuropsychological Battery. Biological Psychiatry, 69, 1192-1203.

Chen, A. C., Feng, W., Zhao, H., Yin, Y., \& Wang, P. (2008). EEG default mode network in the human brain: Spectral regional field powers. Neuroimage, 41, 561-574.

Clarke, A. R., Barry, R. J., Dupuy, F. E., Heckel, L. D., McCarthy, R., Selikowitz, M., \& Johnstone, S. J. (2011). Behavioural differences between EEG-defined subgroups of 
children with attention-deficit/hyperactivity disorder. Clinical Neurophysiology, 122, 1333-1341.

Clarke, A. R., Barry, R. J., Dupuy, F. E., McCarthy, R., Selikowitz, M., \& Johnstone, S. J. (2013). Excess beta activity in the EEG of children with attention-deficit/hyperactivity disorder: A disorder of arousal? International Journal of Psychophysiology, 89, 314-319.

Clarke, A. R., Barry, R. J., McCarthy, R., \& Selikowitz, M. (2001). Age and sex effects in the EEG: Differences in two subtypes of attention-deficit/hyperactivity disorder. Clinical Neurophysiology, 112, 815-826.

Clarke, A. R., Barry, R. J., McCarthy, R., \& Selikowitz, M. (2002). EEG differences between good and poor responders to methylphenidate and dexamphetamine in children with attention-deficit/hyperactivity disorder. Clinical Neurophysiology, 113, 194-205.

Clarke, A. R., Barry, R. J., McCarthy, R., \& Selikowitz, M. (2011). Correlation between EEG activity and behavior in children with attention-deficit/hyperactivity disorder. Journal of Neurotherapy, 15, 193-199.

Clarke, A. R., Barry, R. J., McCarthy, R., Selikowitz, M., Magee, C. A., Johnstone, S. J., \& Croft, R. J. (2006). Quantitative EEG in low-IQ children with attentiondeficit/hyperactivity disorder. Clinical Neurophysiology, 117, 1708-1714.

Cox, C. L., Gotimer, K., Roy, A. K., Castellanos, F. X., Milham, M. P., \& Kelly, C. (2010). Your resting brain CAREs about your risky behavior. PLoS ONE, 5, e12296.

Delorme, A., \& Makeig, S. (2004). EEGLAB: An open source toolbox for analysis of single-trial EEG dynamics including independent component analysis. Journal of Neuroscience Methods, 134, 9-21. doi:10.1016/ j.jneumeth.2003.10.009

Diamond, A. (2013). Executive functions. Annual Review of Psychology, 64, 135-168.

Garrett, D. D., Kovacevic, N., McIntosh, A. R., \& Grady, C. L. (2011). The importance of being variable. Journal of Neuroscience, 31, 4496-4503.

Hermens, D. F., Soei, E. X., Clarke, S. D., Kohn, M. R., Gordon, E., \& Williams, L. M. (2005). Resting EEG theta activity predicts cognitive performance in attention-deficit hyperactivity disorder. Pediatric Neurology, 32, 248-256.

Hsu, C. F., Benikos, N., \& Sonuga-Barke, E. J. (2015). Spontaneous activity in the waiting brain: A marker of impulsive choice in attention-deficit/hyperactivity disorder? Developmental Cognitive Neuroscience, 12, 114-122.

Hsu, C. F., Broyd, S. J., Helps, S. K., Benikos, N., \& Sonuga-Barke, E. J. (2013). "Can waiting awaken the resting brain?" A comparison of waiting- and cognitive task-induced attenuation of very low frequency neural oscillations. Brain Research, 1524, 34-43.

Karam, R. G., Breda, V., Picon, F. A., Rovaris, D. L., Victor, M. M., Salgado, C. A., . . Bau, C. H. (2015). Persistence and remission of ADHD during adulthood: A 7-year clinical follow-up study. Psychological Medicine, 45, 2045-2056.

Koyama, M. S., Di Martino, A., Zuo, X. N., Kelly, C., Mennes, M., Jutagir, D. R., . . . Milham, M. P. (2011). Resting-state functional connectivity indexes reading competence in children and adults. Journal of Neuroscience, 31, 8617-8624.

Larson-Prior, L. J., Power, J. D., Vincent, J. L., Nolan, T. S., Coalson, R. S., Zempel, J., . . . Petersen, S. E. (2011). Modulation of the brain's functional network architecture in the transition from wake to sleep. Progress in Brain Research, 193, 277-294.

Lenartowicz, A., \& Loo, S. K. (2014). Use of EEG to diagnose ADHD. Current Psychiatry Reports, 16(11), Article 498.

Loo, S. K., Cho, A., Hale, T. S., McGough, J., McCracken, J., \& Smalley, S. L. (2013). Characterization of the theta to beta ratio in ADHD: Identifying potential sources of heterogeneity. Journal of Attention Disorders, 17, 384-392. 
Mann, C. A., Lubar, J. F., Zimmerman, A. W., Miller, C. A., \& Muenchen, R. A. (1992). Quantitative analysis of EEG in boys with attention-deficit-hyperactivity disorder: Controlled study with clinical implications. Pediatric Neurology, 8, 30-36.

Mantini, D., Perrucci, M. G., Del Gratta, C., Romani, G. L., \& Corbetta, M. (2007). Electrophysiological signatures of resting state networks in the human brain. Proceedings of the National Academy of Sciences of the United States, 104, 13170-13175.

Miyake, A., Friedman, N. P., Emerson, M. J., Witzki, A. H., Howerter, A., \& Wager, T. D. (2000). The unity and diversity of executive functions and their contributions to complex "frontal lobe" tasks: A latent variable analysis. Cognitive Psychology, 41, 49-100.

Mognon, A., Jovicich, J., Bruzzone, L., \& Buiatti, M. (2011). ADJUST: An automatic EEG artifact detector based on the joint use of spatial and temporal features. Psychophysiology, 48, 229-240.

Nigg, J. T., Willcutt, E. G., Doyle, A. E., \& Sonuga-Barke, E. J. (2005). Causal heterogeneity in attention-deficit/hyperactivity disorder: Do we need neuropsychologically impaired subtypes? Biological Psychiatry, 57, 1224-1230.

Ogrim, G., Kropotov, J., \& Hestad, K. (2012). The quantitative EEG theta/beta ratio in attention deficit/hyperactivity disorder and normal controls: Sensitivity, specificity, and behavioral correlates. Psychiatry Research, 198, 482-488.

Polanczyk, G., de Lima, M. S., Horta, B. L., Biederman, J., \& Rohde, L. A. (2007). The worldwide prevalence of ADHD: A systematic review and metaregression analysis. American Journal of Psychiatry, 164, 942-948.

Putman, P., van Peer, J., Maimari, I., \& van der Werff, S. (2010). EEG theta/beta ratio in relation to fear-modulated response-inhibition, attentional control, and affective traits. Biological Psychology, 83, 73-78.

Raichle, M. E., \& Snyder, A. Z. (2007). A default mode of brain function: A brief history of an evolving idea. Neuroimage, 37, 1083-1090; discussion 1097-1089.

Reineberg, A. E., Andrews-Hanna, J. R., Depue, B. E., Friedman, N. P., \& Banich, M. T. (2015). Resting-state networks predict individual differences in common and specific aspects of executive function. Neuroimage, 104, 69-78.

Roh, S. C., Park, E. J., Park, Y. C., Yoon, S. K., Kang, J. G., Kim, D. W., \& Lee, S. H. (2015). Quantitative electroencephalography reflects inattention, visual error responses, and reaction times in male patients with attention deficit hyperactivity disorder. Clinical Psychopharmacology and Neuroscience, 13, 180-187.

Roh, S. C., Park, E. J., Shim, M., \& Lee, S. H. (2016). EEG beta and low gamma power correlates with inattention in patients with major depressive disorder. Journal of Affective Disorders, 204, 124-130.

Saad, J. F., Kohn, M. R., Clarke, S., Lagopoulos, J., \& Hermens, D. F. (2015). Is the Theta/Beta EEG marker for ADHD inherently flawed? Journal of Attention Disorders.

Satterfield, J. H., \& Cantwell, D. P. (1974). Proceedings: CNS function and response to methylphenidate in hyperactive children. Psychopharmacology Bulletin, 10(4), 36-37.

Sergeant, J. A. (2005). Modeling attention-deficit/hyperactivity disorder: A critical appraisal of the cognitive-energetic model. Biological Psychiatry, 57, 1248-1255.

Shi, T., Li, X., Song, J., Zhao, N., Sun, C., Xia, W., . . . Tomoda, A. (2012). EEG characteristics and visual cognitive function of children with attention deficit hyperactivity disorder (ADHD). Brain \& Development, 34, 806-811.

Snyder, S. M., \& Hall, J. R. (2006). A meta-analysis of quantitative EEG power associated with attention-deficit hyperactivity disorder. Journal of Clinical Neurophysiology, 23, 440-455. 
Snyder, S. M., Quintana, H., Sexson, S. B., Knott, P., Haque, A. F., \& Reynolds, D. A. (2008). Blinded, multi-center validation of EEG and rating scales in identifying ADHD within a clinical sample. Psychiatry Research, 159, 346-358.

Sonuga-Barke, E. J. S. (2005). Causal models of attention-deficit/hyperactivity disorder: From common simple deficits to multiple developmental pathways. Biological Psychiatry, 57, 1231-1238.

Sonuga-Barke, E. J. S., \& Castellanos, F. X. (2007). Spontaneous attentional fluctuations in impaired states and pathological conditions: A neurobiological hypothesis. Neuroscience \& Biobehavioral Reviews, 31, 977-986.

Tabachnick, B. G., \& Fidell, L. S. (2007). Using multivariate statistics (5th ed.). Boston, MA: Pearson/Allyn \& Bacon.

U.S. Food and Drug Administration. (2013). De novo classification request for Neuropsychiatric EEG-Based Assessment Aid for ADHD (NEBA) system. Retrieved from http://www.accessdata.fda.gov/cdrh_docs/reviews/K112711.pdf

van Dongen-Boomsma, M., Lansbergen, M. M., Bekker, E. M., Kooij, J. J., van der Molen, M., Kenemans, J. L., \& Buitelaar, J. K. (2010). Relation between resting EEG to cognitive performance and clinical symptoms in adults with attention-deficit/hyperactivity disorder. Neuroscience Letters, 469, 102-106.

Wang, L., Negreira, A., LaViolette, P., Bakkour, A., Sperling, R. A., \& Dickerson, B. C. (2010). Intrinsic interhemispheric hippocampal functional connectivity predicts individual differences in memory performance ability. Hippocampus, 20, 345-351.

Willcutt, E. G., Doyle, A. E., Nigg, J. T., Faraone, S. V., \& Pennington, B. F. (2005). Validity of the executive function theory of attention-deficit/hyperactivity disorder: A meta-analytic review. Biological Psychiatry, 57, 1336-1346. 